\title{
Correction to: Variability in lutetium-177 SPECT quantification between different state-of-the-art SPECT/CT systems
}

Steffie M. B. Peters ${ }^{1{ }^{*}+}$, Sebastiaan L. Meyer Viol ${ }^{2 \dagger}$, Niels R. van der Werf ${ }^{3 \dagger}$, Nick de Jong ${ }^{4}$, Floris H. P. van Velden ${ }^{4}$, Antoi Meeuwis ${ }^{1}$, Mark W. Konijnenberg ${ }^{3}$, Martin Gotthardt ${ }^{1}$, Hugo W. A. M. de Jong ${ }^{2}$ and Marcel Segbers ${ }^{3}$

The original article can be found online at https://doi.org/10.1186/ s40658-020-0278-3.

* Correspondence: steffie.peters@ radboudumc.nl

${ }^{\dagger}$ Steffie M. B. Peters, Sebastiaan L. Meyer Viol and Niels R. van der Werf contributed equally to this work.

${ }^{1}$ Department of Radiology and Nuclear Medicine, Department of Radiology and Nuclear Medicine, Radboud University Medical Center, P.O. Box 9101, 6500, HB, Nijmegen, The Netherlands

Full list of author information is available at the end of the article

\section{Correction to: EJNMMI Phys 7, 9 (2020)}

https://doi.org/10.1186/s40658-020-0278-3

Following publication of the original article [1], it was reported that the sphere volumes defined in the original article should be adjusted. The correct inner diameters (and volumes) of the spherical inserts were: $9.9 \mathrm{~mm}(0.5 \mathrm{ml}), 15.4 \mathrm{~mm}(2.0 \mathrm{ml}), 19.8$ $\mathrm{mm}(4.0 \mathrm{ml}), 24.8 \mathrm{~mm}(8.0 \mathrm{ml}), 31.3 \mathrm{~mm}(16.0 \mathrm{ml})$ and $60 \mathrm{~mm}(113 \mathrm{ml})$. Figures 3,5 and 6 have been adjusted accordingly.

The original article has been updated.

\section{Author details}

'Department of Radiology and Nuclear Medicine, Department of Radiology and Nuclear Medicine, Radboud University Medical Center, P.O. Box 9101, 6500, HB, Nijmegen, The Netherlands. ${ }^{2}$ Department of Radiology and Nuclear Medicine, University Medical Center Utrecht, Utrecht, The Netherlands. ${ }^{3}$ Department of Radiology and Nuclear Medicine, Erasmus MC, Rotterdam, The Netherlands. ${ }^{4}$ Department of Radiology, Section of Medical Technology, Leiden

University Medical Center, Leiden, The Netherlands.

Published online: 18 August 2021

\section{Reference}

1. Peters SMB, Meyer Viol SL, van der Werf NR, de Jong N, van Velden FHP, Meeuwis A, et al. Variability in lutetium-177 SPECT quantification between different state-of-the-art SPECT/CT systems. EJNMMI Phys. 2020;7:9 https://doi.org/10.11 86/s40658-020-0278-3.
(อ) The Author(s). 2021 Open Access This article is licensed under a Creative Commons Attribution 4.0 International License, which permits use, sharing, adaptation, distribution and reproduction in any medium or format, as long as you give appropriate credit to the original author(s) and the source, provide a link to the Creative Commons licence, and indicate if changes were made. The images or other third party material in this article are included in the article's Creative Commons licence, unless indicated otherwise in a credit line to the material. If material is not included in the article's Creative Commons licence and your intended use is not permitted by statutory regulation or exceeds the permitted use, you will need to obtain permission directly from the copyright holder. To view a copy of this licence, visit http://creativecommons.org/licenses/by/4.0/. 

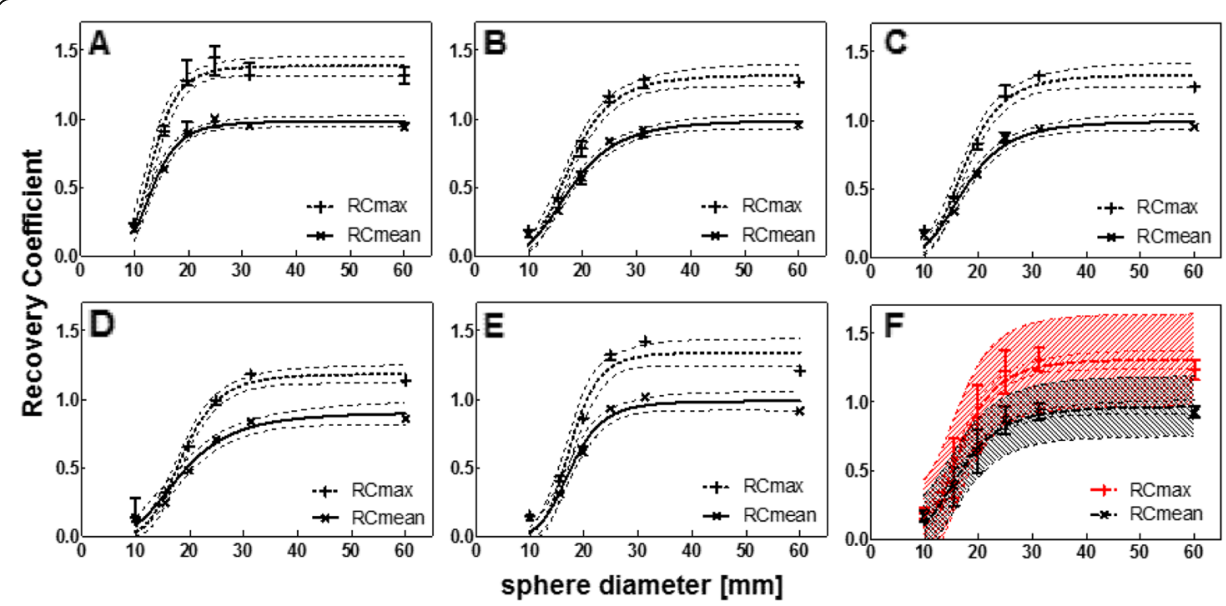

Fig. 3 Recovery coefficient as a function of sphere diameter for all systems separately (A-E) and for all systems combined $(\mathbf{F})$, for data reconstructed with a vendor specific algorithm. Median and range of three repetitive measurements per system. A) Discovery NM/CT 670 Pro; B) Symbia Intevo Bold with XSPECT

Quant; C) Symbia Intevo Bold with Broad Quantification; D) Symbia T16 system 1; E) Symbia T16 system 2; F) Mean and standard deviation. All data were fitted with a 3-parameter logistic function (dashed line: 95\%

$\mathrm{Cl}$ ), for the combined data (F) also the $95 \%$ prediction interval is indicated (dashed area)

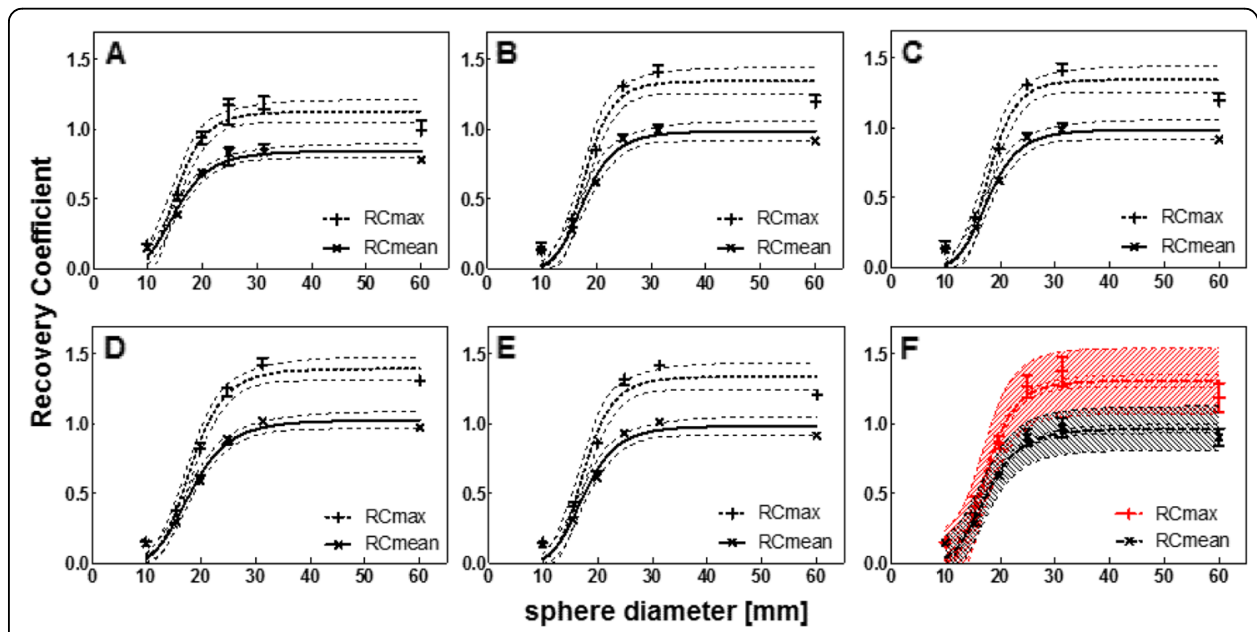

Fig. 5 Recovery coefficient as a function of sphere diameter for all systems separately (A-E) and for all systems combined (F), for data reconstructed with a vendor neutral algorithm. Median and range of three repetitive measurements per system. A) Discovery NM/CT 670 Pro; B) Symbia Intevo Bold with xSPECT Quant; C) Symbia Intevo Bold with Broad Quantification; D) Symbia T16 system 1; E) Symbia T16 system 2; F) Mean and standard deviation for all systems combined. All data were fitted with a 3-parameter logistic function (dashed line: 95\% $\mathrm{Cl}$ ), for the combined data ( $\mathrm{F}$ ) also the $95 \%$ prediction interval is indicated (dashed area 


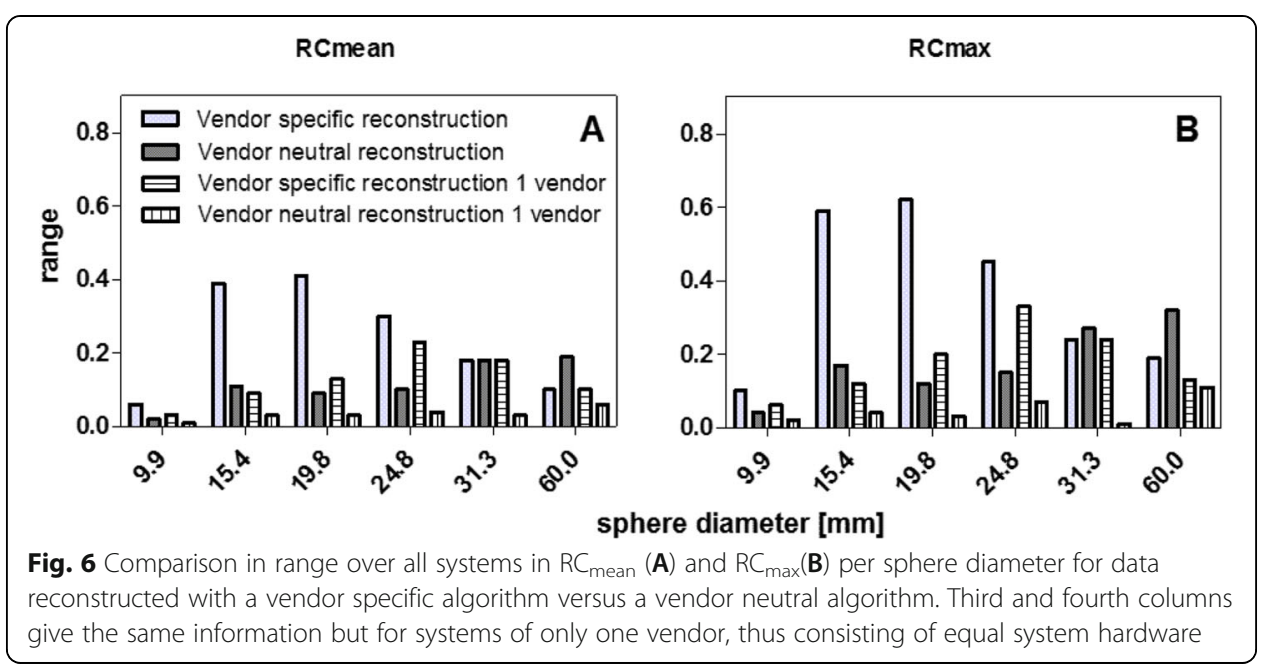

